\title{
Tagging "Dark-Jet" at collider
}

\section{Myeonghun Park}

Institute of Convergence Fundamental Studies and School of Liberal Arts, Seoul National University of Science and Technology, Seoul 01811, Korea

E-mail: parc.seoultecheseoultech.ac.kr

\section{Mengchao Zhang*}

Center for Theoretical Physics of the Universe, Institute for Basic Science (IBS), Daejeon, 34051, Korea

E-mail: mczhang@ibs.re.kr

The phenomenology of dark matter is complicated if dark matter is a composite particle as a hadron under a dark gauge group. Once a dark parton is produced at a high energy collider, it showers and evolves to a jet-like object, eventually it provides a collider signature depending on interactions with particles of the Standard Model (SM). For example, a finite lifetime of dark hadron would provide a displaced vertex. Thus by considering features in various subdetectors, one can identify a jet from a dark parton ("dark jet") with analysis methods in conventional exotic searches. However if the lifetime of the dark hadron is collider-negligible (too short to manifest a displaced vertex), it would be hard to tag a dark jet over Quantum Chromodynamics (QCD) jets of the SM. Thus conventional analyses with information from various sub-detectors are not enough to probe dark matter physics in general at colliders. We propose an analysis to utilize a combination of jet-substructure variables to identify dark jets over backgrounds. We study features of jet-substructure variables for a dark jet. We identify what parameters in dark jet are relevant to performance of a given jet-substructure variable. To maximize performance we apply a boost decision tree (BDT) to jet-substructure variables in tagging dark QCD jet over QCD jets. Our result shows that by combining various jet-substructure variables, one could get a good discrimination performance to identify a dark jet over QCD backgrounds.

The 39th International Conference on High Energy Physics (ICHEP2018)

4-11 July, 2018

Seoul, Korea

${ }^{*}$ Speaker. 


\section{Introduction}

The existence of Dark Matter (DM) in our universe has been confirmed indirectly with its gravitational effects [1]. Still we have no idea about the nature of DM as we have not found DM "directly" with various DM experiments. Especially WIMP (Weakly Interacting Massive Particle) as the most popular DM paradigm has been a subject for various experiments including space indirect searches, nucleon scattering direct searches, and collider experiments. However, we have excluded a wide range in the parameter space of WIMP [2,3] from null results in above searches. So it's natural to consider beyond the WIMP scenario. A popular idea is to introduce a "dark QCD" in the dark sector [4]. The dark gauge group $S U\left(N_{d}\right)$ causes confinement at a certain scale $\Lambda_{d}$, the energetic dark parton, which is the particle charged under $S U\left(N_{d}\right)$, produced in collider will cause a jet-like signal. The feature of this "dark jet" is model dependent. If the lifetime of dark meson is not too short, then it's possible to tag this kind of dark jet by displaced vertex. This kind of dark jet is called "emerging jet" [5]. And if a fraction of light dark meson are stabilized by some symmetry, then there will be missing energy aligned with the jet and transverse mass helps to distinguish the signal. This kind of jet is called "semi-visible jet" [6]. While if the dark mesons inside the dark jet are all unstable and decay promptly, then previous methods will lose their effect. Inspired by quark/gluon jet discrimination in the SM, we try to use jet sub-structure variables to distinguish a promptly decayed dark jet with the SM QCD jet. We will present the dark sector setting and the jet sub-structure variables we chosen in the next section. Then we show our tagging efficiency in section three and conclude in section four.

\section{Dark sector setting and jet sub-structure variables}

In order to cover a range of dark jet with different property, we consider 4 benchmark setting with different confinement scale, meson spectrum, and decay channel. See Tab. 2.

We pick several jet sub-structure variables to do tagging. But only 3 of them are important:

- Two points energy correlation function $C_{1}^{(\beta)}$ [9], which is defined as:

$$
C_{1}^{(\beta)}=\sum_{i<j \in J} z_{i} z_{j}\left(R_{i j}\right)^{\beta}
$$

with $z_{i}=p_{T i} / \sum_{i \in J} p_{T_{i}}$ is the $p_{T}$ fraction carried by component $i$ within a jet $J$, and $R_{i j}$ is the distance between component $i$ and $j$.

- Charged track multiplicity. We count the amount of track with $p_{T}>0.5 \mathrm{GeV}$ inside a jet.

- Energy deposit ratio on different kinds of calorimeters. In our work we call it "E-ratio", and its definition is:

$$
\text { E-ratio }=\frac{\text { Energy deposit on ECAL }}{\text { Jet's } p_{T}} .
$$

The reason of why these variable are useful in tagging dark jet can be found in our paper [10]. 


\begin{tabular}{|l|c|c|c|c|c|c|l|l|}
\hline & $N_{d}$ & $n_{f}$ & $\begin{array}{c}\Lambda_{d} \\
(\mathrm{GeV})\end{array}$ & $\begin{array}{c}\tilde{m}_{q^{\prime}} \\
(\mathrm{GeV})\end{array}$ & $\begin{array}{c}m_{\pi_{d}} \\
(\mathrm{GeV})\end{array}$ & $\begin{array}{c}m_{\rho_{d}} \\
(\mathrm{GeV})\end{array}$ & $\pi_{d}$ Decay Mode & $\rho_{d}$ Decay Mode \\
\hline$A$ & 3 & 2 & 15 & 20 & 10 & 50 & $\pi_{d} \rightarrow c \bar{c}$ & $\rho_{d} \rightarrow \pi_{d} \pi_{d}$ \\
\hline$B$ & 3 & 6 & 2 & 2 & 2 & 4.67 & $\pi_{d} \rightarrow s \bar{s}$ & $\rho_{d} \rightarrow \pi_{d} \pi_{d}$ \\
\hline$C$ & 3 & 2 & 15 & 20 & 10 & 50 & $\begin{array}{l}\pi_{d} \rightarrow \gamma^{\prime} \gamma^{\prime} \text { with } \\
m_{\gamma^{\prime}}=4.0 \mathrm{GeV}\end{array}$ & $\rho_{d} \rightarrow \pi_{d} \pi_{d}$ \\
\hline$D$ & 3 & 6 & 2 & 2 & 2 & 4.67 & $\begin{array}{l}\pi_{d} \rightarrow \gamma^{\prime} \gamma^{\prime} \text { with } \\
m_{\gamma^{\prime}}=0.7 \mathrm{GeV}\end{array}$ & $\rho_{d} \rightarrow \pi_{d} \pi_{d}$ \\
\hline
\end{tabular}

Table 1: Models we considered in this work. All dark hadrons are assumed to decay promptly. We mainly consider 2 cases: high $\Lambda_{d}$ case like A and C, low $\Lambda_{d}$ case like B and D. Parameters in a dark sector for A and $\mathrm{C}, \mathrm{B}$ and $\mathrm{D}$ are the same except the decay channel of a dark pion $\pi_{d} . \pi_{d}$ and $\rho_{d}$ mass obey following two equations: $m_{\pi_{d}}=2 \tilde{m}_{q^{\prime}}-\frac{3}{4} \frac{\Omega}{\tilde{m}_{q^{\prime}}^{2}}$ and $m_{\rho_{d}}=2 \tilde{m}_{q^{\prime}}+\frac{1}{4} \frac{\Omega}{\tilde{m}_{q^{\prime}}^{2}}$ [7]. Here $\tilde{m}_{q^{\prime}}$ is constituent dark quark mass and parameter $\Omega$ can be determined by other input parameters. The ratio of $\pi_{d}$ to $\rho_{d}$ after fragmentation is decided by $\frac{\# \pi_{d}}{\# \rho_{d}}=\frac{1}{3} e^{-\frac{m \pi_{d}-m \rho_{d}}{\Lambda_{d}}}$. The branching ratios of their decay modes shown here are all $100 \%$, if we don't give a specific value. Decay modes of a dark photon $\gamma^{\prime}$ with different mass can be found in [8].

\section{Tagging efficiency}

To cut away a high density region in multi-dimensional profile of background QCD jet made with various jet-substructure variables, we use Boosted Decision Tree (BDT) [11] in TMVAToolkit [12]. We use 500 decision trees, choose minimum in leaf node as $2.5 \%$, and set maximum depth as 3. To avoid overtraining, half of the events are chosen as test events and KolmogorovSmirnov test is required to be larger than 0.01. In Fig. 3 we show the final tagging results for different model setting and different variable combination.
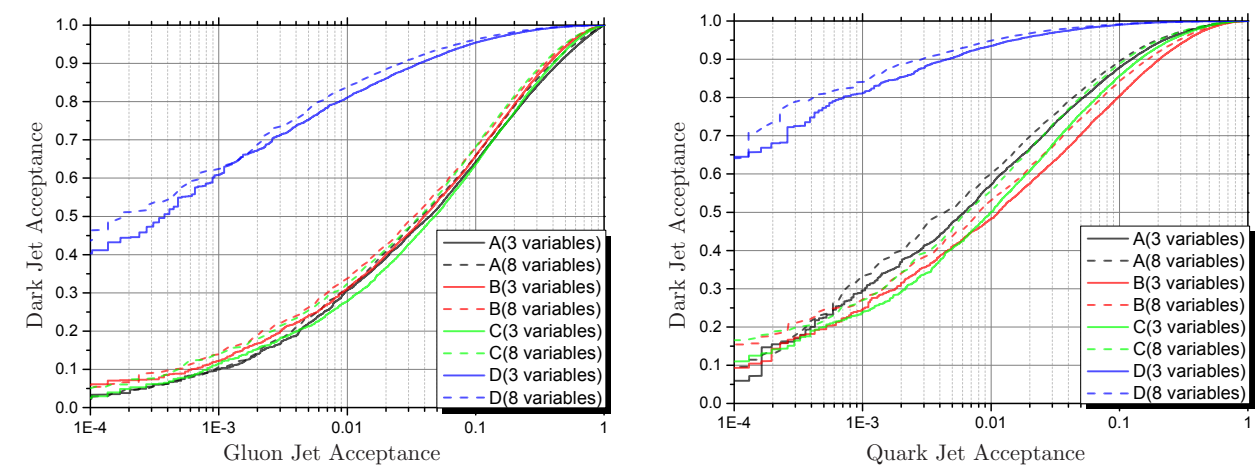

Figure 1: ROC curves from BDT. Left: dark jet vs. gluon jet ROC curves for all four models and different variables combination, with $p_{T} \in(180 \mathrm{GeV}, 220 \mathrm{GeV})$. Right: dark jet vs. quark jet ROC curves for all four models and different variables combination, with $p_{T} \in(180 \mathrm{GeV}, 220 \mathrm{GeV}) .3$ variables means a combination of $\left\{C_{1}^{(\beta)}\right.$, E-ratio, Track Multiplicity $\}$. 3 variables means a combination of $\left\{m_{J} / p_{T}, C_{1}^{(\beta)}\right.$, Girth, Track Multiplicity, E-ratio, number of sub-jets, $\left.f_{p_{T}}^{(1)}, f_{p_{T}}^{(2)}\right\}$. 


\section{Conclusion}

Dark sector under a strong interaction provides composite states and corresponding attractive phenomenologies. A large theoretical degrees of freedom of this scenario lead to a diverse and model dependent phenomenology. At colliders, such model introduce jet-like signal (called "dark jet"), some of which may not be tagged by distinct or exotic signatures including missing energy or displaced vertex. In this work, inspired by the success of quark/gluon jet discrimination, we try to distinguish dark jet from background SM QCD-jet by using jet sub-structure variables. A series of jet sub-structure variables, like the jet mass, $C_{1}^{(\beta)}$, or track multiplicity, are used to discriminate dark jets from QCD-jets. Combination of these variables with boosted decision tree (BDT) shows a great discriminant performance. For all of our model settings and jet's $p_{T} \gtrsim 200 \mathrm{GeV}$, we can exclude $99 \%$ background gluon jets while reserving more than $30 \%$ signal dark jet, or exclude 99\% background quark jet while reserving more than 50\% signal dark jet. Our results demonstrate that by considering the information inside a jet, we will get a much better understanding of dark jet and enhance collider search sensitivity to identify signatures of dark QCD model at the LHC.

\section{References}

[1] P. A. R. Ade et al. [Planck Collaboration], "Planck 2015 results. XIII. Cosmological parameters," Astron. Astrophys. 594, A13 (2016) doi:10.1051/0004-6361/201525830 [arXiv:1502.01589 [astro-ph.CO]].

[2] A. Tan et al. [PandaX-II Collaboration], "Dark Matter Results from First 98.7 Days of Data from the PandaX-II Experiment,” Phys. Rev. Lett. 117, no. 12, 121303 (2016) doi:10.1103/PhysRevLett.117.121303 [arXiv:1607.07400 [hep-ex]].

[3] D. S. Akerib et al. [LUX Collaboration], "Results from a search for dark matter in the complete LUX exposure,” Phys. Rev. Lett. 118, no. 2, 021303 (2017) doi:10.1103/PhysRevLett.118.021303 [arXiv:1608.07648 [astro-ph.CO]].

[4] Y. Bai and P. Schwaller, Phys. Rev. D 89, no. 6, 063522 (2014) doi:10.1103/PhysRevD.89.063522 [arXiv:1306.4676 [hep-ph]].

[5] P. Schwaller, D. Stolarski and A. Weiler, “Emerging Jets,” JHEP 1505, 059 (2015) doi:10.1007/JHEP05(2015)059 [arXiv:1502.05409 [hep-ph]].

[6] T. Cohen, M. Lisanti and H. K. Lou, "Semivisible Jets: Dark Matter Undercover at the LHC," Phys. Rev. Lett. 115, no. 17, 171804 (2015) doi:10.1103/PhysRevLett.115.171804 [arXiv:1503.00009 [hep-ph]].

[7] D. Griffiths, "Introduction to elementary particles," Weinheim, Germany: Wiley-VCH (2008) $454 \mathrm{p}$

[8] M. Buschmann, J. Kopp, J. Liu and P. A. N. Machado, "Lepton Jets from Radiating Dark Matter," JHEP 07, 045 (2015) doi:10.1007/JHEP07(2015)045 [arXiv:1505.07459 [hep-ph]].

[9] A. J. Larkoski, G. P. Salam and J. Thaler, “Energy Correlation Functions for Jet Substructure,” JHEP 1306, 108 (2013) doi:10.1007/JHEP06(2013)108 [arXiv:1305.0007 [hep-ph]].

[10] M. Park and M. Zhang, arXiv:1712.09279 [hep-ph].

[11] B. P. Roe, H. J. Yang, J. Zhu, Y. Liu, I. Stancu and G. McGregor, Nucl. Instrum. Meth. A 543, no. 2-3, 577 (2005) doi:10.1016/j.nima.2004.12.018 [physics/0408124].

[12] A. Hocker et al., PoS ACAT , 040 (2007) [physics/0703039 [PHYSICS]]. 